\title{
Challenging times for environmental health in South Africa: The role of the Environmental Health Research Network
}

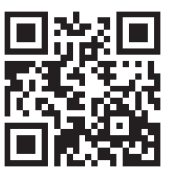

Environmental health aims to create environments supportive of good health and to minimise disease. This is achieved by controlling environmental factors that impact adversely on human health. Throughout Africa, modern environmental health hazards, including mercury, lead, air toxins, water contaminants, pesticides, and domestic and hazardous waste ${ }^{[1]}$ need to be addressed alongside longstanding environmental health concerns such as poor housing, inadequate water and sanitation, and exposure to indoor air pollution from the use of solid and liquid fuels for cooking.

In South Africa (SA), public protection against environmental hazards is addressed in Section 24 of the Constitution, which states that everyone has the right to an environment that is not harmful to their health and wellbeing, while Section 27 upholds the right of access to healthcare services and sufficient food and water. Responsibility for the provision of safe and healthy environments in which communities live, learn and play is predominantly vested in the local sphere of government. Local-level environmental (or municipal) health services include water quality monitoring, food control and waste management, vector control, environmental pollution control, surveillance and prevention of contagious diseases, and disposal of the dead. Environmental health practitioners (EHPs) form the backbone of environmental health services in SA, and face a significant challenge to provide the safe and healthy environment all South Africans need to maximise their life potential.

\section{High-level challenges impeding environments for healthy lives}

The key environmental health challenges ${ }^{[2]}$ that need to be overcome are closely linked to the history of SA, continuing poverty and inequality ${ }^{[3]}$ as well as over-consumption. The underlying reasons for current environmental health challenges are complex and fundamental change is necessary to address them. The country also has to grapple with major international forces affecting the environment and health status, such as globalisation and rapid urbanisation, unmatched by optimal levels of service delivery. The upshot is that SA is faced with a quadruple burden of disease including HIV/AIDS, violence and injuries, and communicable and non-communicable diseases. ${ }^{[4]}$
While progress has undoubtedly been made in environmental health since the advent of democracy, in many respects environmental health policy and legislation in SA is not always clear, and there is sometimes a disconnection between national and local roles and responsibilities. Policy and legislation are also covered by several different Acts, agreements and conventions, ${ }^{[3]}$ making it difficult to address the growing range of environmental health issues efficiently. Transformation of the environmental health service in SA remains incomplete; for example, devolution of environmental health to local government is unfinished in several provinces. Also, while compulsory community-based environmental health service has been instituted as part of training for EHPs, the systems and funding are not always in place to accommodate all trainees, unfairly rendering them unable to graduate or accept work appointments. There is also a well-established shortage of EHPs in the country, with the ratio of EHPs to population falling well below international guidelines (Cele A, 'Strengthening the provision of MHS within the country' - unpublished paper presented at the Municipal Health Conference, Pretoria, 20 June 2013).

Over and above current environmental health problems, climate change and how it affects temperature, water and air poses another significant challenge in maintaining environmental quality for human health, although adaptation options open the door for innovative solutions beneficial to environmental health.

\section{A trichotomous structure in environmental health}

Environmental health may be considered in three parts: governance, the profession and research. The National Department of Health (NDoH)'s Environmental Health Directorate is responsible for governing environmental health operations and procedures in SA, but it is woefully under-capacitated. The Department oversees and implements environmental health operations and procedures via the EHPs, who function within the parameters of a 'scope of practice' document at local government level. EHPs are registered with the Health Professions Council of SA, and the South African Institute of Environmental Health (affiliated with the International Federation of Environmental Health) serves as their professional organisation. 
Despite the shortcomings outlined here, mechanisms are in place with regard to governance and operations in environmental health. Where the co-ordination of environmental health research is concerned, however, little is in place. To date no national state of environmental health report has been written for SA. An underlying reason for this is the fragmentation of research data and information across multiple institutions and documents, making the collation of relevant data and the preparation of a coherent report a mammoth undertaking. The need for a repository for SA environmental health research and the linking of researchers in this domain has therefore arisen.

\section{Multi-disciplinary, co-ordinated research and partnerships}

In 2009, the Environmental Health Research Network (EHRN) ${ }^{[5]}$ was launched. The NDoH's Environmental Health Director, Ms Aneliswa Cele, endorsed the EHRN at an inaugural seminar in April 2010. The overall aim of the EHRN is to serve as a network to share knowledge and ideas about environmental health research. It is a 'community of practice' in environmental health research in SA. The network is open to academic and research institutions, government departments, non-government organisations (NGOs) and any other interested parties. The principal objective of the EHRN is to develop and co-ordinate research, information and practical resources on environmental health matters at all levels. The EHRN aims to provide networking opportunities to everyone working in any environmental health research field to consolidate existing research and identify gaps in research. The purpose of the network is threefold: (i) to strengthen environmental health research in SA; (ii) to encourage collaboration and idea sharing in environmental health research; and (iii) to gain a better understanding of the state of environmental health in SA.

The EHRN is an assembly of people working in environmental health research across different organisations, institutions and agencies. It is an open forum for discussion, and currently comprises $>150$ members from SA. While most members are researchers and scientists working in environmental health research, EHPs, NGOs and government officials make frequent use of the network to learn more about environmental health research in SA. In 2013, the EHRN bi-monthly lecture series was initiated, in which a prominent scientist prepares lecture materials related to a topical environmental health issue. Lectures are distributed electronically to the EHRN membership. A further core activity of the EHRN is to motivate for the development of environmental health indicators and the collection of environmental health data at appropriate levels of aggregation and scale, linked to socio-demographic data to support evidence-based research and policy making.

The vision of the EHRN is to be the primary point of contact for all those who are interested in environmental health research in SA. It aims to facilitate multidisciplinary research, leading to a more thorough description of the state of environmental health in SA and providing a basis to evaluate change. Within a context of limited capacity and resources, the platform provided by the EHRN is fundamental to environmental health research in this country, and to the strategies, planning and decisions necessary to grapple with current and emerging environmental health challenges, especially those posed by the advent of climate change.

\section{Y Wright}

Climate Studies, Modelling and Environmental Health Research Group, Council for Scientific and Industrial Research, Pretoria, South Africa

\section{A Mathee}

Environment and Health Research Unit, South African Medical Research Council, Johannesburg, South Africa

\section{A Oosthuizen}

Climate Studies, Modelling and Environmental Health Research Group, Council for Scientific and Industrial Research, Pretoria, South Africa

\section{Corresponding author: C Y Wright (cwright@csir.co.za)}

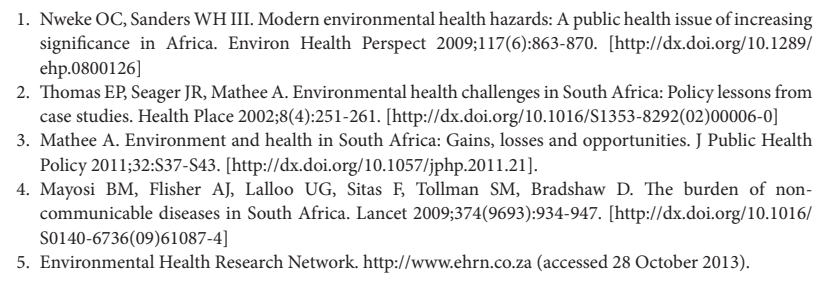

